University of Nebraska - Lincoln

DigitalCommons@University of Nebraska - Lincoln

Gordon Gallup Publications

Research Papers in Physics and Astronomy

May 2001

\title{
Quantum-Mechanical Analysis of a Longitudinal Stern-Gerlach Effect
}

Gordon A. Gallup

UNL,ggallup1@unl.edu

Herman Batelaan

University of Nebraska - Lincoln, hbatelaan@unl.edu

Timothy J. Gay

University of Nebraska - Lincoln, tgay1@unl.edu

Follow this and additional works at: https://digitalcommons.unl.edu/physicsgallup

Part of the Physics Commons

Gallup, Gordon A.; Batelaan, Herman; and Gay, Timothy J. , "Quantum-Mechanical Analysis of a Longitudinal Stern-Gerlach Effect" (2001). Gordon Gallup Publications. 39.

https://digitalcommons.unl.edu/physicsgallup/39

This Article is brought to you for free and open access by the Research Papers in Physics and Astronomy at DigitalCommons@University of Nebraska - Lincoln. It has been accepted for inclusion in Gordon Gallup Publications by an authorized administrator of DigitalCommons@University of Nebraska - Lincoln. 


\title{
Quantum-Mechanical Analysis of a Longitudinal Stern-Gerlach Effect
}

\author{
G. A. Gallup, H. Batelaan, and T. J. Gay \\ Behlen Laboratory of Physics, University of Nebraska, Lincoln, Nebraska 68588-0111
}

(Received 28 August 2000)

\begin{abstract}
We present the results of a rigorous quantum-mechanical calculation of the propagation of electrons through an inhomogeneous magnetic field with axial symmetry. A complete spin polarization of the beam is demonstrated assuming that a Landau eigenstate can be inserted into the field. This is in contrast with the semiclassical situation, where the spin splitting is blurred.
\end{abstract}

DOI: $10.1103 /$ PhysRevLett.86.4508

PACS numbers: $34.80 . \mathrm{Nz}, 03.65 . \mathrm{Ta}, 14.60 . \mathrm{Cd}$

The Stern-Gerlach experiment is one of the most important in the history of physics and is often used to illustrate the nature of spin in quantum mechanics [1]. Curiously, a magnet of the type used by Stern and Gerlach does not work with beams of electrons because of the combined effects of the Lorentz force and the Heisenberg uncertainty principle. This was shown first by Mott and Bohr in 1928 [2]. Pauli subsequently made a more general argument that no device based on the concept of classical particle trajectories and macroscopic magnetic fields could be used to separate an electron beam by spin or to measure the electron's magnetic moment [3]. The Bohr/Mott/Pauli argument is codified in numerous textbooks [4].

In this Letter we show that, in fact, it is possible to observe spin splitting of a beam of electrons using a longitudinal magnetic-field configuration instead of the standard transverse geometry of Stern and Gerlach. The longitudinal configuration has the advantage that the electrons experience only off-axis Lorentz forces that are significantly smaller than the on-axis forces in the transverse geometry. Such an idea was first proposed by Brillouin [5], but was specifically rejected by Pauli. Recently, however, we discovered an error in the reasoning Pauli used against Brillouin's idea, and analyzed a counterexample using classical particle trajectories in which spin splitting of electrons could be achieved equal to the blurring caused by the Lorentz forces [6-8]. Although these results are intriguing, they do not take into account the wave nature of the electron, and thus do not address the central question: Can a spin separation really be expected? In this article we report the results of a rigorous quantum-mechanical analysis of the longitudinal Stern-Gerlach problem, which corresponds to physical reality. We obtain the surprising result that complete separation can be achieved, an improvement over the semiclassical situation. Complete spin splitting is thus shown to be, fundamentally, a quantum-mechanical effect.

It is clear that the magnetic moment of individual electrons can be measured; Dehmelt and his colleagues accomplished this with a modified Penning trap [9]. Separation of electrons by spin does not follow from these experiments, however, even though Pauli used the same general argument against both. Quantum calculations have shown spin separations in some static situations [10,11]. For the beam configuration considered by Pauli et al., however, the situation is more ambiguous. Adler [12] and Garroway and Stenholm [13] verified Pauli's analysis of the transverse-field geometry. More importantly for this discussion, Bloch [14] and Dehmelt [9] sketched quantum arguments for longitudinal-field geometries and suggested that complete isolation of the lowest energy "spin-backward" state should be possible. They did not, however, consider the nondestructive case of full transmission of both spin components in a beam. An experiment of the "Bloch-Dehmelt" type was performed by Knight and his colleagues in the mid 1960s, and observation of a low energy $\left(<10^{-8} \mathrm{eV}\right)$ tail of (presumably) spin-polarized electrons was reported [15]. This work was never formally published, and, apparently, could not be reproduced [9]. Sannikov [16] and Conte et al. [17] considered the problem we take up here, i.e., longitudinal spatial spin separation of a fully transmitted beam, but did not use electron wave packets having extended transverse dimensions. This precludes any elimination of the splitting due to blurring of the separate spin states.

Our calculation begins with the full nonrelativistic Hamiltonian for an electron in the magnetic field $\vec{B}(\rho, \phi, z)$ of a simple current ring. The ring lies in the $x-y$ plane, has radius $R$, and is centered at the origin. Thus,

$$
\begin{aligned}
\vec{B}(\rho, \phi, z)= & -\frac{1}{2} \rho \frac{d B(z)}{d z}[\cos (\phi) \hat{x}+\sin (\phi) \hat{y}] \\
& +B(z) \hat{z}
\end{aligned}
$$

where

$$
B(z)=B_{0}\left(\frac{R}{\sqrt{R^{2}+z^{2}}}\right)^{3},
$$

and $B_{0}>0$ is the field at the origin assuming $\rho / R \ll$ 1. Schrödinger's equation for this problem in cylindrical coordinates about the $z$ axis is

$$
\left[H_{L}(z)+H_{z}\right] \psi=i \hbar \frac{\partial \psi}{\partial t},
$$




$$
\begin{aligned}
H_{L}(z)= & -\frac{\hbar^{2}}{2 m_{e}} \frac{1}{\rho} \frac{\partial}{\partial \rho}\left(\rho \frac{\partial}{\partial \rho}\right)+\frac{L_{z}^{2}}{2 m_{e} \rho^{2}} \\
& +\omega_{L}(z)\left(L_{z}+g S_{z}\right)+\frac{1}{2} m_{e}\left[\omega_{L}(z)\right]^{2} \rho^{2},
\end{aligned}
$$

$$
H_{z}=-\frac{\hbar^{2}}{2 m_{e}} \frac{\partial^{2}}{\partial z^{2}}+g \frac{d \omega_{L}}{d z}\left(x S_{x}+y S_{y}\right),
$$

where $\omega_{L}(z)$ is the Larmor frequency $\left(\frac{|e B(z)|}{2 m_{e} c}\right), L_{z}$ is the operator for the canonical angular momentum of the electron about the $z$ axis, $S_{x}, S_{y}$, and $S_{z}$ are the Cartesian spin operators, $m_{e}$ is the electron mass, and $g=2\left(1+a_{e}\right)$ is the anomalous electron gyromagnetic ratio. Equations (4) and (5) bear some discussion. The first two terms of (4) and the first term of (5) correspond to the electron's kinetic energy. In (4), both the fourth term and the part of the third term involving $L_{z}$ correspond to the classical $-\vec{\mu}_{\text {orb }} \cdot \vec{B}$ potential of an electron with orbital angular momentum about $z$. The latter part of the third term in (4) and the second term in (5) are the equivalent terms for the magnetic potential associated with electron spin. If $\vec{B}$ were uniform, the last term of (5) would vanish, and both $m_{l}$ and $m_{s}$, the quantum numbers associated with $L_{z}$ and $S_{z}$, would separately be good. Generally, for the Hamiltonian of (3), only $m_{j}=m_{l}+m_{s}$ is a good quantum number.

We now construct complete wave packet solutions of the form

$$
\psi=\sum_{n} \sum_{m_{l}} \sum_{m_{s}} R_{n}^{\left(m_{l}\right)}(\rho, \phi) \eta_{m_{s}} a_{n m_{l} m_{s}}(z, t),
$$

where $\eta$ is a spinor, the $a_{n m_{l} m_{s}}(z, t)$ are functions that contain all of the explicit $z$ and $t$ dependence of $\psi$ as the electrons move along the magnetic field, and

$$
H_{L} R_{n}^{\left(m_{l}\right)} \eta_{m_{s}}=E_{n m_{l} m_{s}} R_{n}^{\left(m_{l}\right)} \eta_{m_{s}} .
$$

Note that the $R_{n}^{\left(m_{l}\right)}(\rho, \phi)$ have a parametric dependence upon $z$, but otherwise are solutions to the standard Landau problem. They span $x-y$ space and can be written as

$$
\begin{aligned}
R_{n}^{\left(m_{l}\right)}(\rho, \phi)= & N_{n\left|m_{l}\right|}(\sqrt{\alpha} \rho)^{\left|m_{l}\right|} L_{n}^{\left(\left|m_{l}\right|\right)}\left(\alpha \rho^{2}\right) \\
& \times \exp \left(-\frac{1}{2} \alpha \rho^{2}\right) e^{i m_{l} \phi}, \\
L_{n}^{\left(\left|m_{l}\right|\right)}(x)= & \frac{(-1)^{n}}{n ! x^{\left|m_{l}\right|}} e^{x} \frac{d^{n}}{d x^{n}}\left[x^{n+\left|m_{l}\right|} e^{-x}\right], \\
\alpha= & \alpha(z)=\frac{m \omega(z)}{\hbar},
\end{aligned}
$$

and

$$
N_{n\left|m_{l}\right|}=\sqrt{\frac{n ! \alpha}{\pi\left(n+\left|m_{l}\right|\right) !}},
$$

where

$$
E_{n m_{l} m_{s}}=\hbar \omega_{L}(z)\left(2 n+\left|m_{l}\right|+m_{l}+g m_{s}+1\right) .
$$

In order to leave $m_{l}$ as an explicit quantum number, we have not followed the common practice of denoting $2 n+$ $\left|m_{l}\right|$ as another single integer.

Substituting (6) into (3) and using (7), we obtain a set of coupled equations for the $\alpha_{n m_{l} m_{s}}$. The coupling terms in these equations determine how nonadiabatic the electron transmission process is, i.e., how likely it is for an electron in a given Landau uniform-field eigenstate to depart from that state over the course of its passage through the magnetic field. We estimate these terms by using the physical quantities considered in the semiclassical calculation of Ref. [6]. The electrons follow a $2 \mathrm{~m}$ path length that has a midpoint at the center of a $2 \mathrm{~cm}$ radius current ring, where the field magnitude $B_{0}$ is $10 \mathrm{~T}$. Their initial speed is taken to be $10^{5} \mathrm{~m} / \mathrm{s}(=28 \mathrm{meV})$. These values yield a reasonable splitting $(631 \mu \mathrm{m})$ of the spin in a semiclassical model for on-axis trajectories. From the point of view of this paper, the $10 \mathrm{~T}$ maximum field means that magnetic potential energies for the electron along the trajectory will always be $\ll 28 \mathrm{meV}$ for the lowest Landau levels.

The above experimental conditions justify two approximations. First, the $z$ dependence of the Landau Hamiltonian [Eq. (4)] could cause transitions between uniform-field Landau eigenstates. We find that these off-diagonal coupling terms can be neglected with one exception: those associated with the magnetic-field gradient that arise from the second term on the right of Eq. (5), corresponding to electron spin flip. Second, given that the magnetic potential energies are much smaller than the electron kinetic energy, the WKB approximation is valid for the longitudinal wave function propagation.

The spin-flip probability can be characterized by the ratio of coupling terms $\mathrm{U}$ :

$$
\left|\frac{U_{i j}}{U_{i i}-U_{j j}}\right|=\frac{3(z / R)}{4 a_{e} \sqrt{\alpha_{0}} R\left[1+(z / R)^{2}\right]^{1 / 4}},
$$

where $i$ and $j$ label states connected by the transverse spin operator. Although not negligible, this quantity is $<10^{-2}$ at all values of $z$, and corresponds to spin-flip probabilities of the same order of magnitude. Thus the electrons traverse the magnetic field almost completely adiabatically; if a wave packet that corresponds to a Landau eigenfunction can be inserted into the field, the probability is high that it will emerge in the same state. It is interesting to note that the small amount of spin flipping that does occur is inversely proportional to the electron $g$-factor anomaly.

We now consider the transmission of the two Landau wave packets with $n, m_{l}, m_{s}=0,0, \pm 1 / 2$. These states are most strongly coupled to the $0, \pm 1, \mp 1 / 2$ states, respectively, but this coupling is negligible, as discussed above. The packets are superpositions of plane waves such that 


$$
\begin{aligned}
a_{00 \pm 1 / 2}(z, t=0) \equiv & a_{ \pm}(z, 0) \\
= & (2 \pi \hbar)^{-1 / 2} \int \phi\left(p_{z}\right) \\
& \times \exp \left(i p_{z} z / \hbar\right) d p_{z},
\end{aligned}
$$

where $\phi\left(p_{z}\right)$ is taken to be Gaussian-like with a spatial width along $z$ of $\chi_{0}^{2}$ and a momentum spread of $\hbar / \sqrt{2 \chi_{0}}$. At later times,

$$
\begin{aligned}
a_{ \pm}(z, t)= & (2 \pi \hbar)^{-1 / 2} \int \phi(p) \\
& \times \exp \left(i\left[\frac{p z}{\hbar}+\delta_{ \pm}(E)-\frac{E t}{\hbar}\right]\right) d p,
\end{aligned}
$$

where we have used the WKB approximation for

$$
\delta_{ \pm}(E)=2 \sqrt{\alpha_{0}} R G\left(\frac{E}{\left(2 \pm a_{e}\right) \hbar \omega_{0}}\right)
$$

and

$$
G(x)=\sqrt{x} \int_{-\infty}^{\infty}\left[\left(1-\frac{1}{x\left(1+\xi^{2}\right)^{3 / 2}}\right)^{1 / 2}-1\right] d \xi .
$$

The value of $\chi_{0}^{2}$ should be chosen to minimize the spreading of the wave packet along $\hat{\mathbf{z}}$ over the electron flight time $l / v_{0}$. This condition yields

$$
\chi_{0, \min }=\sqrt{\frac{\hbar l}{m_{e} v_{0}}}
$$

and corresponds to $80 \mu \mathrm{m}$ in the present case.

The WKB phase shift can be expanded as

$$
\begin{aligned}
\delta_{ \pm}(p)= & \delta_{0}+\delta_{1, \pm}\left(p-p_{0}\right)+(1 / 2) \delta_{2}\left(p-p_{0}\right)^{2} \\
& +\ldots
\end{aligned}
$$

and interpreted as follows: the wave packet is displaced by a distance $\hbar \delta_{1, \pm}$ relative to its position in the absence of a magnetic potential, and spreads by an amount corresponding to the normal spreading of a free wave packet at time $t$ plus an extra amount corresponding to an additional time increment, $m_{e} \hbar \delta_{2}$.

The results of our calculation for the "spin-forward" and "spin-backward" minimum uncertainty wave packets are shown in Fig. 1. Their most striking feature is the virtually complete separation of the two packets, in marked contrast with our previous calculations [6]. In those calculations, "magnetic bottle" forces associated with the mechanical orbital angular momentum of the electron "smeared" both packets by an amount equal to their centroid splitting. In the present situation, the individual wave packet spreading is essentially that which one would observe in a field-free measurement, with a very small additional spreading characterized by $\delta_{2}$. The lack of spreading, when compared with the semiclassical case, results because the eigenenergies of the electron wave packet depend not on the mechanical angular momentum but on the canonical angular momentum $L_{z}$, which is sharp. Since the electron wave packet is characterized by a sharp eigenenergy, the only

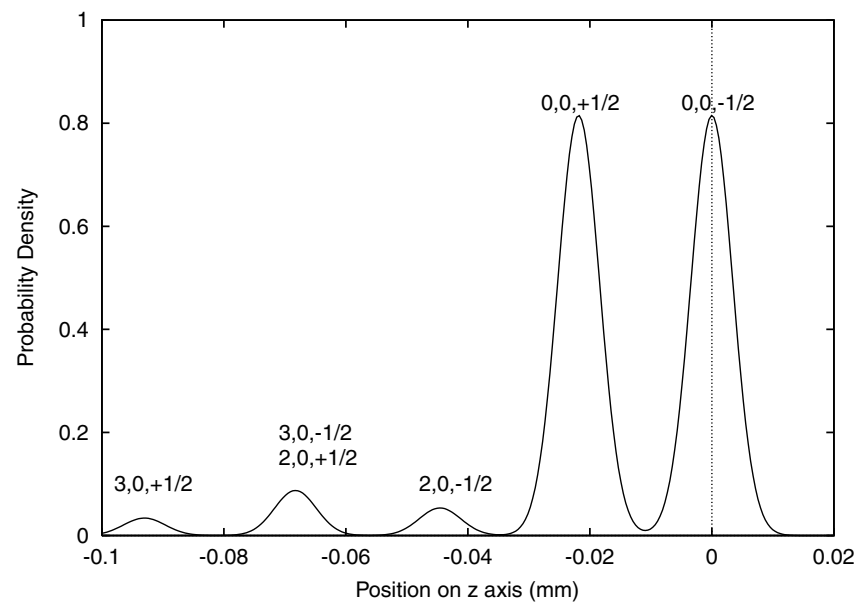

FIG. 1. Electron probability density vs distance along the $z$ axis after traversal of a $2 \mathrm{~m}$ flight path. Each peak is marked with its principal $\left(n, m_{l}, m_{s}\right)$ values. Distance indicated is the deviation from the position of the leading packet, which equals the field-free position. The $n=2$ and $n=3$ peaks are contaminant contributions caused by the insertion of the electron into the magnetic field (see text).

relevant "force" acting on it is proportional to the longitudinal spatial derivative of the magnetic potential (and thus $\partial B / \partial z$ ) and is therefore sharp as well. The complete spin splitting is thus seen to be, fundamentally, a quantummechanical effect.

A quantitative measurement of the spin splitting can be made using the parameter $F=S / W$, where $W$ is the full width at half maximum of one of the two spin components along the $z$ axis, and $S$ is the splitting distance separating the two spin component centroids. Thus $F=0$ in the field-free case, unity if the spins are just resolved using Rayleigh's criterion, and $\gg 1$ for "complete" splitting. $F$ is plotted in Fig. 2 as a function of mean electron energy $E_{0}$. Wave packets obeying the minimum spreading criterion have widths at the end of a given field-free path length $l$ proportional to $E_{0}^{-1 / 4}$, whereas the spin splitting varies as $E_{0}^{-1}$. Thus, even at the lowest energies we consider, magnetic splitting always dominates natural wave packet spreading.

The initial packets must have the spatial dimensions and angular momentum properties of the ground Landau states in a minimum-spreading longitudinal configuration. This means that they must be cylindrically symmetric so that $m_{l}=0$. Assuming an energy of $28 \mathrm{meV}$, minimum spreading requires a longitudinal velocity uncertainty of $1 \mathrm{~m} / \mathrm{s}$ and a pulse duration of $2 \mathrm{ps}$. We assume that the electron beam is defined by two (or more) circular apertures along the symmetry axis of the current ring. The insertion of Landau eigenstates into the $B$-field region occurs at a final aperture in, e.g., a highly permeable boundary wall. It can be shown that this aperture's diameter should be $d=\sqrt{20 h / e B}$, where $B$ is the field several diameters, $d$, inside the container. This value of $d$ ensures 


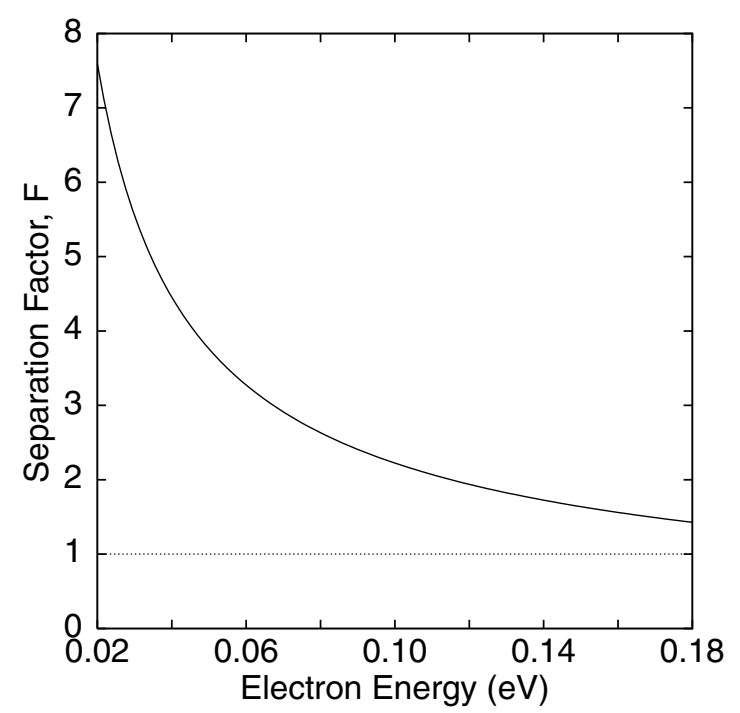

FIG. 2. Spin splitting figure of merit $F$ vs electron energy. When $F=1$, the opposite-spin wave packets just meet the Rayleigh resolution criterion.

maximum overlap between the $a_{00 \pm 1 / 2}$ states and an incident wave whose transverse dimensions are much larger than the aperture. In this case, electrons emerging into the magnetic-field region have an $82 \%$ probability of being in the $n=0$ state. The chance of being in a state with $n<16$ is $95 \%$. The effect of these "contaminant" $m_{l}=0$ states is shown in Fig. 1.

For an apparatus whose typical dimension is $1 \mathrm{~m}, B$ will be of the order of a Gauss at the entrance aperture $(d=5 \mu)$. The size of the aperture is large enough to prevent appreciable diffraction as the electrons enter the magnetic-field region; their de Broglie wavelength at this energy is $<10^{-8} \mathrm{~m}$. Finally we note that the magnetic field "leaks" out into the low-field region from the entrance aperture with an inverse third power spatial dependence on the beam axis [18]. This results in negligible distortion of the electron plane waves as they approach the aperture.

It appears there is no fundamental physics preventing the observation of spin splitting. We speculate that the most pernicious problem for the realization of our thought experiment would be the nonideal nature of real collimating apertures, manifesting itself in spurious electric and magnetic-field effects. A better experimental approach may well be one of the type discussed by Conte et al. [17], in which the beam to be polarized is that of a synchrotron, and the separation is effected through a series of longitudinal Stern-Gerlach "kicks."

The authors thank Emil Sidky and Marlan Scully for useful conversations. This work was supported in part by NSF Grant No. PHY-9702350, Research Corp. Grant No. RI0380, and TIAA-CREF.

[1] W. Gerlach and O. Stern, Z. Phys. 9, 349 (1922).

[2] N. F. Mott, Proc. R. Soc. London A 124, 425 (1929).

[3] W. Pauli, in Magnetism (Gauthier-Villars, Brussels, 1930), pp. 217-226.

[4] See, e.g., C. Cohen-Tannoudji, B. Diu, and F. Laloe, Quantum Mechanics (Wiley, New York, 1977), p. 969; E. Merzbacher, Quantum Mechanics (Wiley, New York, 1970), 2nd ed., p. 254; N. F. Mott and H. S. W. Massey, The Theory of Atomic Collisions (Clarendon Press, Oxford, 1965), 3rd ed., p. 214; H.C. Ohanian, Principles of Quantum Mechanics (Prentice-Hall, Englewood Cliffs, NJ, 1990), p. 243; J. Kessler, Polarized Electrons (Springer-Verlag, Berlin, 1985), 2nd ed., p. 2; Quantum Theory and Measurement, edited by J. A. Wheeler and W. H. Zurek (Princeton University Press, Princeton, NJ, 1983), p. 701; G. Baym, Lectures on Quantum Mechanics (Benjamin, New York, 1969), p. 328.

[5] L. Brillouin, Proc. Natl. Acad. Sci. U.S.A. 14, 755 (1928).

[6] H. Batelaan, T. J. Gay, and J. J. Schwendiman, Phys. Rev. Lett. 79, 4517 (1997).

[7] G. H. Rutherford and R. Grobe, Phys. Rev. Lett. 81, 4772 (1998).

[8] H. Batelaan and T. J. Gay, Phys. Rev. Lett. 81, 4773 (1998).

[9] H. Dehmelt, Z. Phys. D 10, 127 (1988).

[10] H. Martens and W. M.d. Muynck, J. Phys. A 26, 2001 (1993).

[11] G. H. Rutherford and R. Grobe, J. Phys. A 31, 9331 (1998).

[12] F. Adler, Helv. Phys. Acta 10, 455 (1937).

[13] B. M. Garraway and S. Stenholm, Phys. Rev. A 60, 63 (1999).

[14] F. Bloch, Physica 19, 821 (1953).

[15] L. V. Knight, in Physics (Stanford University Press, Palo Alto, CA, 1965), p. 97.

[16] S. S. Sannikov, Sov. Phys. JETP 19, 1186 (1964).

[17] M. Conte, A. Penzo, and M. Pusterla, Nuovo Cimento 108A, 127 (1995).

[18] J. D. Jackson, Classical Electrodynamic (Wiley, New York, 1975), 2nd ed., pp. 125-127. 